\title{
INFECCIÓN NATURAL POR Trypanosomacruzi EN TRIATOMINOS QUE HABITAN EN LA PALMA COROZO (Acrocomia aculeta) EN REGIONES DEL ORIENTE DE VENEZUELA
}

\author{
Antonio Morocoima ${ }^{1, a}$, Rogny Barroeta ${ }^{1, b}$, Milagros Virguez $^{1, b}$, Antonio Roschman-González ${ }^{2, \mathrm{c}}$, \\ José David Chique ${ }^{1, d}$, Elizabeth Ferrer $^{3, e}$, Leidi Herrera ${ }^{4, f}$
}

\begin{abstract}
RESUMEN
Objetivos. Conocer la infestación natural por triatominos y su infección por Trypanosoma cruzi (T. cruzi) en Acrocomia aculeata (A. aculeata) o palma corozo en el estado Anzoátegui, Venezuela. Materiales y métodos. Se estudió la infestación triatomínica y su infección por $T$. cruzi en $A$. aculeata desafectadas en campañas fitosanitarias. La presencia del parásito se determinó por microscopia y PCR-kDNA, y se realizó su caracterización mediante marcadores moleculares. Resultados. Se encontraron 14 palmeras con infestación triatomínica, el 48,8 \% de los ejemplares correspondieron a Rhodnius prolixus y el $48,2 \%$ a Triatoma maculata, con desarrollo ontogénico hacia el adulto. Las pruebas parasitológicas y moleculares, su morfología típica y la infección en el modelo murino revelaron la presencia de $T$. cruzi en $54,8 \%$ en promedio, para ambas especies de triatominos, con circulación del genotipo Tcl de T. cruzi. Conclusiónes. Se reportó para el estado Anzoátegui en Venezuela, la infestación de palma corozo con Rhodnius prolixus y Triatoma maculata y la presencia de subpoblaciones Tcl de T. cruzi, siendo esta palma el hábitat peridomiciliar del binomio triatominos-T. cruzi y posible bioindicador de riesgo de infección para poblaciones humanas circunvecinas.
\end{abstract}

Palabras claves: Triatominae; Trypanosoma cruzi; Venezuela (fuente: DeCS BIREME)

\section{TRIATOMINAE IN PALM TREES AND THEIR NATURAL INFECTION BY Trypanosomacruzi IN REGIONS OF EASTERN VENEZUELA}

\begin{abstract}
Introduction. To know the natural infestation by triatominae and their infection by Trypanosoma cruzi (T. cruzi) in Acrocomia Aculeata (A. aculeata) or coyol palm in the state of Anzoátegui, Venezuela. Materials and Methods. Triatominic infestation and its infection by $\mathrm{T}$. cruzi was studied in non-affected A. aculeata in phytosanitary campaigns. The presence of the parasite was determined by microscopy and PCR-kDNA, and its characterization was made by means of molecular markers. Results. Fourteen palm trees with triatominic infestation were found; $48.8 \%$ of the individuals corresponded to Rhodnius prolixus and $48.2 \%$ to Maculata Triatoma, with ontogenetic development towards adult. The parasitology and molecular tests, their typical morphology and the infection in the murine model revealed the presence of $T$. cruzi in an average of $54,8 \%$, for both species of triatominae, with circulation of the Tcl genotype of $T$. cruzi. Conclusions. The infestation of coyol palm trees with Rhodnius prolixus and Maculata Triatoma was reported for the state of Anzoátegui in Venezuela, as well as the presence of Tcl sub-populations of T. cruzi, being this palm tree the peridomicilar habitat of the triatominae-T. cruzi binomial and possible bioindicador of risk of infection for surrounding human populations.
\end{abstract}

Keywords: Triatominae; Trypanosoma cruzi; Venezuela (source: MeSH NLM)

Centro de Medicina Tropical, Universidad de Oriente (UDO). Anzoátegui, Venezuela.

Centro de Microscopia Electrónica; Facultad de Ciencias, Universidad Central de Venezuela (UCV). Caracas, Venezuela.

Instituto de Investigaciones Biomédicas «Dr. Francisco J. Triana Alonso» (BIOMED), Universidad de Carabobo Sede Aragua, Las Delicias. Maracay, Venezuela.

Centro de Ecología y Evolución, Instituto de Zoología y Ecología Tropical (IZET), Facultad de Ciencias, Universidad Central de Venezuela (UCV). Caracas, Venezuela.

Médico cirujano, MSc en Parasitología; ${ }^{\mathrm{b}}$ médico cirujano; ${ }^{\mathrm{c}}$ licenciado en Biologia, doctor en Ciencias-Zoologia; ${ }^{\mathrm{d}}$ técnico de laboratorio ${ }^{\dagger} ;{ }^{\mathrm{e}}$ licenciada en Bioanálisis, doctora en Biología Molecular; ${ }^{\mathrm{f}}$ licenciada en Biólogia, doctora en Ciencias -Biología Parasitaria

Recibido: 20/08/2018 Aprobado: 05/12/2018 En línea: 21/12/2018

El estudio procede de una tesis «Estudio de infestación de Acrocomia aculeata Linneo (Palmae) por triatominos e infección natural por Trypanosoma cruzi en vectores capturados, zona noreste y central del estado Anzoátegui» para optar al título de médico cirujano del autor Rogny Barroeta, al cual se le añadió el estudio molecular de la caracterización de Trypanosoma cruzi para su publicación.

Citar como: Morocoima A, Barroeta R, Virguez M, Roschman-González A, Chique JD, Ferrer E, et al. Infección natural por Trypanosoma cruzi en triatominos que habitan en la palma corozo (Acrocomia aculeta) en regiones del oriente de Venezuela. Rev Peru Med Exp Salud Publica. 2018;35(4):563-72.doi: 10.17843/ rpmesp.2018.354.3871 


\section{INTRODUCCIÓN}

Trypanosoma cruzi (T. cruzi) (Excavata, Kinetoplastea, Trypanosomatidae) agente etiológico de la enfermedad de Chagas afecta entre seis y ocho millones de personas en Latinoamérica, donde hay también 25 millones de personas en riesgo, por estar en simpatría con áreas de distribución geográfica de los insectos vectores y mamíferos reservorios ${ }^{(1)}$.

En Venezuela, hay seis millones de personas en riesgo para la infección por T. cruzi, en el pie de monte occidental (Trujillo, Lara, Portuguesa y Barinas) son comunes las viviendas típicas de palma y bahareque; en los estados centrales (Carabobo, Aragua, Distrito capital y Miranda) existen pequeños focos en viviendas rurales y focos totalmente urbanos. Recientemente los estados orientales (Anzoátegui, Monagas, Nueva Esparta) son considerados áreas vulnerables ${ }^{(2-4)}$.

Los triatominos vectores de T. cruzi, ocupan más de 12 ecótopos naturales en el Neotrópico entre los cuales las palmeras representan uno de los más antiguos, estables y de más amplia distribución, siendo fuente de dispersión de triatominos hacia el peridomicilio y domicilio, por su cercanía y/o por el uso de las hojas de palmeras en la construcción de corrales de aves, caneyes, techos de la vivienda o por el consumo de sus frutos por el hombre ${ }^{(2,5,6)}$.

Entre las especies de palmas reportadas con infestación triatomínica se cuentan a Attalea humboldtiana (palma de agua); Copernitia tectorum (palma llanera), Acrocomia aculeata o Acrocomia sclerocarpa (palma corozo), Coccus nucifera (palma de coco) y Mauritia minor (palma moriche) ${ }^{(2,4,5)}$, lo cual ha llevado a las alcaldías de algunos estados del oriente de Venezuela a desafectar ejemplares a menos de 10 metros de las viviendas.

Se realizó un estudio descriptivo para conocer la infestación triatomínica de Acrocomia aculeata desafectadas en campañas fitosanitarias en áreas endémicas de la enfermedad de Chagas en el estado Anzoátegui en Venezuela y la infección natural de estos insectos por T. cruzi, así como la identificación de los genotipos circulantes del parásito; a fin de poder determinar si esta palma es un bioindicador de riesgo para la enfermedad de Chagas en poblaciones humanas.

\section{MATERIALES Y MÉTODOS}

\section{ÁREA DE ESTUDIO}

El área de estudio correspondió a los municipios Simón Bolívar (Parroquia Bergantín: caseríos el Zamuro, La Maravilla y Paraminal) a 310 msnm y ubicado a $10^{\circ} 00^{\prime} 58.31 "$

\section{MENSAJES CLAVE}

Motivación para realizar el estudio. Se propuso describir los elementos presentes en la palma corozo a fin de establecer si representan o no un elemento de riesgo para la dispersión de triatominos y el establecimiento de la enfermedad de Chagas en el oriente venezolano.

Principales hallazgos. Se encontraron 14 palmas corozo o Acrocomia aculeata con infestación por Rhodnius prolixus y Triatoma maculata, en todos sus estadios, infectados con el genotipo TcI de Trypanosoma cruzi, agente etiológico de la enfermedad de Chagas.

Implicancias. Las palmeras constituyen fuentes de triatominos cerca del domicilio, pudiendo ser un factor de riesgo para el establecimiento de la enfermedad de Chagas.

N - 64²2'00.86" O; Municipio Santa Ana (Caserío Santa Gertrudis) a $173 \mathrm{msmn}$ y ubicado 9²0'15" N - 64'15'10" O y Municipio Aragua de Barcelona (Caserío El Ventorillo) a 103 msnm y ubicado a $9^{\circ} 27^{\prime} 27^{\prime \prime} \mathrm{N}-64^{\circ} 49^{\prime} 34^{\prime \prime} \mathrm{O}$, todos del estado Anzoátegui, el cual ocupa el 4,7\% del territorio nacional con 1453274 habitantes y una densidad de 32,41 habitantes por $\mathrm{km}^{2}(6,7)$. La variabilidad climática de la región se debe a factores altitudinales, continentales y marítimos, con altas temperaturas durante todo el año $\left(27^{\circ} \mathrm{C}\right.$ en promedio anual). El periodo más caluroso se ubica entre marzo y mayo y el de lluvias desde mayo a octubre. La precipitación promedio varía entre los $700 \mathrm{~mm}^{3}$ y los $1400 \mathrm{~mm}^{3}$ (Figura 1).

\section{SELECCIÓN DE LAS PALMERAS Y DETERMINACIÓN DE SU INFESTACIÓN TRIATOMÍNICA}

Por ocurrencia de una campaña gubernamental regional de urgente desafectación de palmas, para control de invasión triatomínica en las viviendas humanas, se propuso un estudio descriptivo para conocer la infestacion en los ejemplares desafectados de los cuales se tomaron muestras foliares de $A$. aculeata (Acrocomia totai o Acrocomia sclerocarpa syn; Palmae), conocida como palma corozo de amplia distribución, desde México hasta Argentina, incluyendo las Antillas ${ }^{(5,9)}$.

Los criterios de inclusión fisionómica para ejemplares de palmas desafectados, ubicados en un transepto de 100 metros, mostrar cualitativamente vitalidad y vigor reflejados en una altura de madurez vegetal entre nueve a 20 metros, copa de tres metros de diámetro, estípites de dos a tres centímetros de diámetro. Otros elementos de vitalidad, considerados para la inclusión fueron la existencia de una corteza lisa y oscura con espinas fuertes y rectas, hojas perennes pinnadas, con folíolos en planos diferentes, raquis duro y espinoso de 1,5 - 3,5 metros de largo, cogollos vitales, presencia de inflorescencias en espádices de color amarillo pálido o pardo y de drupas racimosas, todo ello en función de poder mantener una fauna endógena ${ }^{(9)}$. Las palmeras fueron desafectadas entre abril de 2011 a septiembre de 2012 . 


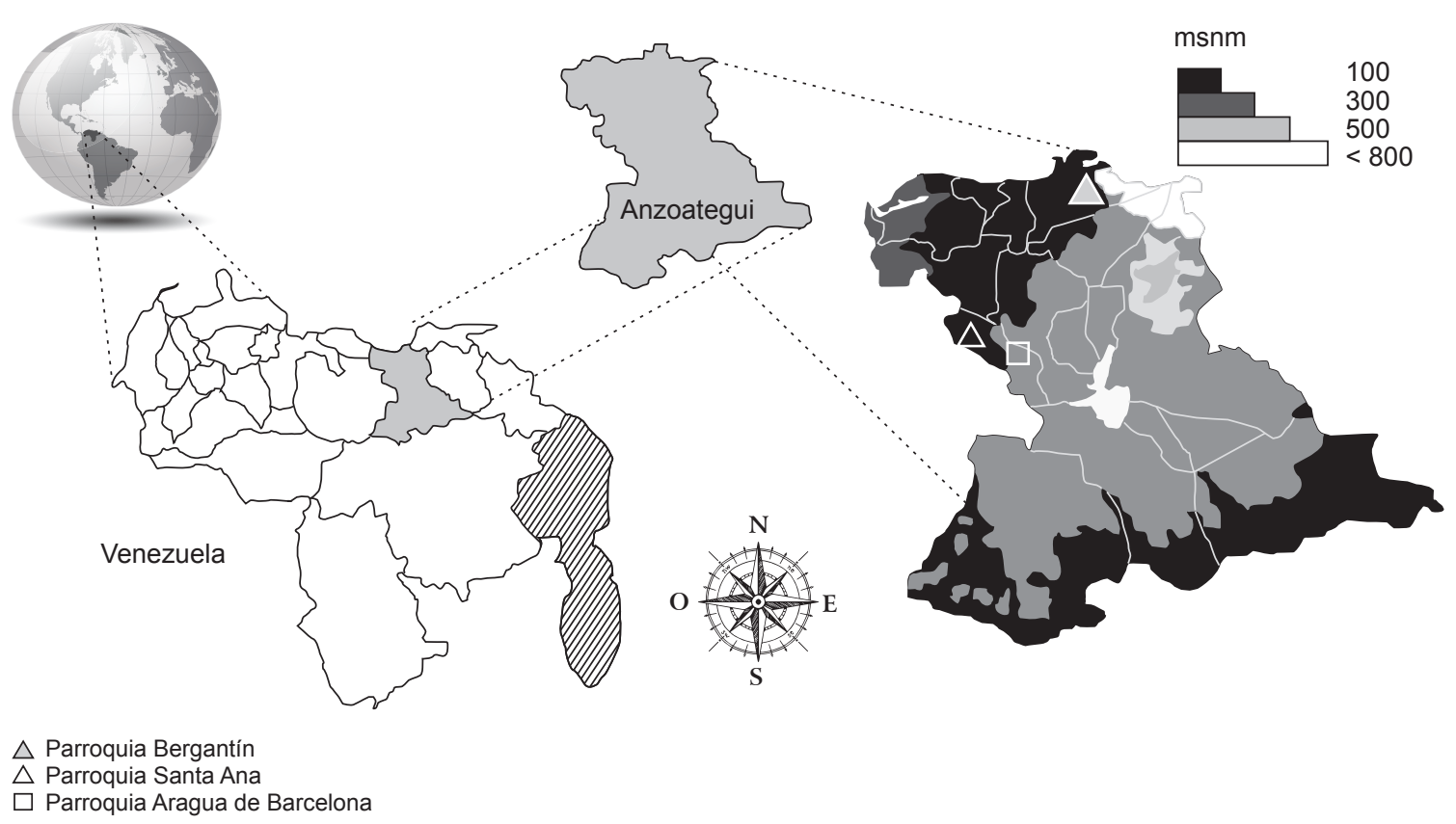

Figura 1. Mapa del área de estudio para la revisión de ejemplares de Acrocomia aculeata del estado Anzoátegui, Venezuela

Los elementos foliares fueron manualmente disecados en forma no destructiva según Gamboa ${ }^{(9)}$, con un esfuerzo de cinco horas/hombre/vegetal para cinco hombres por día, entre las 9 am y 4 pm, conducente a la búsqueda de estadios ontogénicos de triatominos en hojas secas y verdes, en los detritos húmedos de las axilas, en la malla interfoliar y en las brácteas. Todo el material fue examinado sobre mantas blancas para la mejor visión de la entomofauna presente y conducido para el laboratorio debidamente rotulado para separación e identificación de triatóminos con claves biológicas tanto para adultos como para ninfas ${ }^{(10)}$.

Los estadios ninfales recogidos y debidamente rotulados, por palma, fueron mantenidos en condiciones de insectario, a temperatura controlada $\left(28 \pm 2{ }^{\circ} \mathrm{C}\right)$, humedad relativa de $70 \%$ y fotoperiodo de 12 horas. La alimentación de estos estadios ninfales se realizó cada 11 días, sobre gallina durante 30 minutos para promover su desarrollo ontogénico y llegar a estado adulto, para la identificación y asociación de cada estadio de la especie ${ }^{(10)}$

\section{EXAMEN DE LOS TRIATOMINOS PARA LA BÚSQUEDA E IDENTIFICACIÓN DE Trypanosoma}

Para la determinación de infección por Trypanosoma, el intestino de cada triatomino fue extraído y su contenido fue diluido en solución salina fisiológica estéril (0,9 \%) para examen microscópico (400X). El material contentivo de flagelados morfológicamente compatibles con Trypanosoma fue inoculado en lotes de tres ratones NMRI (Naval Medical Research Institute) machos de 10 gramos de peso corporal, a razón de 200 metacíclicos del contenido intestinal/gramo de peso, determinados por el método de Brener modificado según protocolo de baja virulencia (11), para seguimiento de infección y certificación del parásito.

La búsqueda de estadios compatibles con Trypanosoma rangeli, se realizó examinando hemolinfa obtenida de la sección parcial de los tarsos anteriores y/o posteriores y disección de glándulas salivares. El contenido intestinal de los triatominos que presentaron flagelados fue evaluado para aislar ADN total utilizando la resina Chelex® 100 (BioRad), según instrucciones del fabricante. La concentración y pureza del ADN fue establecida ${ }^{(12)}$.

La presencia de ADN de T. cruzi fue determinada mediante la amplificación por PCR (Polimerase Chain Reaction) de la secuencia altamente repetitiva del ADN de los minicírculos del kinetoplasto (ADNk), usando los cebadores 121 (5'-AAATAATGTACGGG(T/G) GAGATGCATGA3') y 122 (5' GGTTCGATTGGGGTTGGTGTAATATA3'). La reacción de amplificación fue realizada con un volumen final de $25 \mu \mathrm{L}$, utilizando buffer GoTaq Flexi (Promega) (Tris HCl 50 mM, pH 9.0; $\left.\mathrm{NaCl}_{2} 50 \mathrm{mM}\right), \mathrm{MgCl}_{2} 2 \mathrm{mM}$, Oligos 121 y $122(0,4 \mu \mathrm{M})$, dNTPs 0,2 mM, ADN polimerasa GoTaq Flexi (Promega) $1 U$ y ADN molde $10 \mu \mathrm{L}$. El programa de amplificación se llevó a cabo en un termociclador (C1000 BioRad) con desnaturalización inicial a $94{ }^{\circ} \mathrm{C}$ por 10 minutos, 30 ciclos de desnaturalización a $94^{\circ} \mathrm{C}$ por 1 minuto, hibridación a $63^{\circ} \mathrm{C}$ por 1 minuto, extensión a $72^{\circ} \mathrm{C}$ por 1 minuto y extensión final a $72^{\circ} \mathrm{C}$ por 10 minutos ${ }^{(12,13)}$. Los productos de amplificación de 330 pares de bases $(\mathrm{pb})$ indicaron la presencia de ADN de $T$. cruzi, certificando genéticamente al parásito. 
La caracterización molecular de los aislados de T. cruzi obtenidos de los triatominos fue realizada usando los siguientes marcadores moleculares: (i) la región intergénica de del espaciador no transcrito para el gen de mini exón usando los cebadores TC 5'-CCCCCCTCCCAGGCCACACTG-3', TC1 5'-GTGTCCGCCACCTCCTTCGGGCC-3' у TC2 5'CCTGCAGGCACACGTGTGTGTG-3'; (ii) el dominio divergente D7 de la fracción 24Sa del gen ribosomal, empleando los cebadores: D71, 5'-AAGGTGCGTCGACAGTGTGG-3' y D72 5'-TTTTCAGAATGGCCGAACAGT-3' y (iii) el dominio de tamaño variable de la fracción $18 \mathrm{~S}$ del gen ribosomal usando el cebador: V1, 5'-CAAGCGGCTGGGTGGTTATTCCA-3' and V2, 5'-TTGAGGGAAGGCATGACACATGT-3'. Para todos estos marcadores moleculares la reacción de amplificación incluyó tampón para la Taq polimerasa $(100 \mathrm{mM}$ Tris- $\mathrm{HCl}$, pH 8.3), 0,2 mM de solución de dNTPs, 1,5 mM de solución de $\mathrm{MgCl}$, $1 \mathrm{U}$ of GoTaq® Flexi DNA Polymerase (Promega, Madison, USA), 0,5 $\mu \mathrm{M}$ de cada iniciador, $10 \mu \mathrm{L}$ de ADN molde y agua libre de nucleasas hasta llegar a $25 \mu \mathrm{L}$ de volumen final. Los ciclos de amplificación fueron establecidos ${ }^{(14)}$ y los productos de PCR para cada reacción fueron analizados por electroforesis en gel de agarosa al $2 \%$ y visualizados mediante tinción con bromuro de etidio.

\section{ANÁLISIS DE LOS DATOS}

Siendo la palma la unidad de análisis y que los modelos de estudios de presencia y progresión de estadios ontogénicos de triatóminos siguen una distribución unimodal, la variabilidad de la infección por T. cruzi en todos los estadios ontogénicos para ambas especies de triatóminos se analizó mediante la prueba de ANOVA de dos vías con $95 \%$ de confianza, a fin de comparar el promedio de porcentajes de infección entre las especies de triatóminos registrados y entre sus estados ontogénicos. Las relaciones entre las características fisionómicas de las palmas y la infestación triatomínica se exploraron mediante el estudio de coeficientes de correlación. Se aceptó un valor de $p<0,05$ como estadísticamente significativo.

\section{RESULTADOS}

Se analizaron 14 palmeras desafectadas, caracterizadas por la presencia de penacho compuesto de hasta 130 hojas pinnadas y proyecciones espinosas oscuras en cada peciolo, altura promedio del árbol de nueve metros, doble proporción de hojas frescas en relación a las hojas secas y un promedio de cuatro racimos de drupa/palmera, todo independientemente de la región geográfica. Los ejemplares se correspondieron fisionómicamente con la especie $A$. aculeata (Tabla 1).

En los caseríos de la zona noreste se encontró el $57 \%$ de palmas corozo muestreadas con $100 \%$ de infestación triatomínica. En todas las palmeras se hallaron huevos eclosionados con una media aproximada de cuatro huevos eclosionados/palma y una media de 12 adultos/palma independientemente de la especie de triatómino.

Huevos y adultos fueron localizados en detritos de las axilas de las pencas, en las «barbas» o «cerdillas» de los bordes de las pencas o «conchas» y en la superficie, interna de estas. Los huevos no eclosionados fueron conducidos al laboratorio y colocados en estufa a $28{ }^{\circ} \mathrm{C}$ y a $75 \%$ de humedad relativa, eclosionando, en promedio, luego de dos semanas y media a tres semanas, resultando en ejemplares viables que alcanzaron el estadio adulto de la especie.

Tabla 1. Características fisionómicas vegetales e infestación triatomínica por huevos y adultos en Acrocomia aculeata de cinco caseríos rurales del estado Anzoátegui, Venezuela

\begin{tabular}{|c|c|c|c|c|c|c|c|}
\hline Caserío & $\begin{array}{l}\text { Número } \\
\text { de palma }\end{array}$ & $\begin{array}{c}\text { Altura } \\
\text { (metros) }\end{array}$ & $\begin{array}{l}\text { Hojas secas } \\
\text { (n) }\end{array}$ & $\begin{array}{l}\text { Hojas verdes } \\
\text { (n) }\end{array}$ & $\begin{array}{l}\text { Racimos } \\
\text { (n) }\end{array}$ & $\begin{array}{c}\text { Huevos } \\
\text { eclosionados (n) }\end{array}$ & $\begin{array}{l}\text { Triatominos } \\
\text { adultos (n) }\end{array}$ \\
\hline \multirow[t]{3}{*}{ El Zamuro } & 1 & 11 & 18 & 44 & 6 & 4 & 10 \\
\hline & 2 & 10 & 28 & 32 & 2 & 5 & 25 \\
\hline & 3 & 9 & 10 & 26 & 3 & 2 & 17 \\
\hline \multirow[t]{3}{*}{ Paraminal } & 4 & 9 & 21 & 32 & 5 & 4 & 5 \\
\hline & 5 & 10 & 12 & 42 & 4 & 10 & 9 \\
\hline & 6 & 14 & 19 & 38 & 2 & 20 & 12 \\
\hline \multirow[t]{2}{*}{ La Maravilla } & 7 & 12 & 8 & 37 & 4 & 3 & 10 \\
\hline & 8 & 11 & 12 & 35 & 4 & 7 & 28 \\
\hline \multirow[t]{4}{*}{ Santa Gertrudis } & 9 & 12 & 10 & 36 & 5 & 0 & 15 \\
\hline & 10 & 11 & 9 & 43 & 4 & 0 & 11 \\
\hline & 11 & 13 & 18 & 24 & 3 & 0 & 16 \\
\hline & 12 & 15 & 20 & 36 & 5 & 0 & 8 \\
\hline \multirow[t]{2}{*}{ Ventorrillo } & 13 & 17 & 20 & 38 & 6 & 0 & 0 \\
\hline & 14 & 15 & 17 & 35 & 4 & 0 & 0 \\
\hline Promedio & - & 12 & 16 & 35 & 4 & 4 & 12 \\
\hline
\end{tabular}


En los caseríos de la zona central se encontró el $47 \%$ de las palmas corozo, con un $67 \%$ (4/6) de infestación triatomínica, el total de palmeras infestadas correspondieron al caserío «Santa Gertrudis», las cuales presentaron todos los estadios ninfales para ambas especies a excepción de huevos. Las palmeras estudiadas en el caserío «Ventorrillo» no presentaron infestación triatomínica.

El índice de infestación triatomínica de las palmeras fue de $85,7 \%$ (12/14) con un total de 166 ejemplares, discriminados 81 como Rhodnius prolixus $(48,8 \%)$ y 80 como Triatoma maculata $(48,2 \%)$, para un promedio de 12 triatominos por palma. Las ninfas de primer estadio para ambas especies se encontraron en las costras húmedas de las axilas de las pencas, compartiendo el hábitat con alacranes, grillos, ciempiés, cucarachas, tuqueques y esporádicamente roedores, marsupiales y quirópteros. Las otras fases evolutivas se encontraron distribuidas en el resto de la planta (Tablas 1 y 2 ).

Para ambas especies de triatominos, la mayoría de sus estadios ontogénicos se encontraron en número creciente a medida que avanzaba hacia el estadio adulto, a excepción de las ninfas de quinto estadio que en todos los casos mostraron descenso en número.

Una fuerte correlación positiva $(r=0,95)$ entre la abundancia de hojas verdes (24 a 44 hojas) en las palmeras y la infestación por adultos sin discriminar por especie y/o de la época de estudio fue encontrada.

El estadio predominante para Rhodnius prolixus fue el adulto $(30 / 86=35 \%)$, seguido por el IV estadio ninfal $(24 / 86$ $=28 \%$ ), en tanto que para Triatoma maculata el IV estadio ninfal $(31 / 80=39 \%)$ fue el predominante en relación al adulto $(23 / 80=29 \%)$ (Tabla 2, Figura 2$)$.

Del total de triatominos colectados (166), se encontró un índice de infección por flagelados compatibles con Trypanosoma en $54,8 \%(91 / 166)$, todos observados al examen microscópico del contenido intestinal en fresco. Un 60,5\% (52/86) de los triatóminos infectados correspondieron a Rhodnius prolixus y el $48,8 \%(39 / 80)$ a Triatoma maculata, siendo relativamente mayor el porcentaje de infección para Rhodnius prolixus, pero sin diferencias significativas $(\mathrm{F}=2,83, \mathrm{p}=0,09)$.

Al analizar el porcentaje de infección entre los estadios del desarrollo ontogénico se apreciaron diferencias significativas $(F=10,90, p<0,01)$. El estadio con mayor porcentaje de infección fue el adulto para $R$. prolixus y IV estadio para $T$. maculata (Tabla 2, Figura 2).

En los triatominos capturados, no se observaron flagelados en hemolinfa y macerados de glándulas salivares, descartándose la presencia de Trypanosoma rangeli.

Tabla 2. Número de ejemplares y su porcentaje de infección con Trypanosoma cruzi discriminados por estadio ontogénico de Triatoma maculata y Rhodnius prolixus en Acrocomia aculeata de caseríos del estado Anzoátegui, Venezuela

\begin{tabular}{|c|c|c|c|c|c|c|c|c|c|c|c|c|c|c|c|c|c|c|}
\hline \multirow[t]{2}{*}{ Palma } & \multicolumn{7}{|c|}{$\begin{array}{c}\text { Estadios de } \\
\text { Triatoma maculata }\end{array}$} & \multicolumn{2}{|c|}{$\begin{array}{l}\text { Infección } \\
\text { por T. cruzi }\end{array}$} & \multicolumn{7}{|c|}{$\begin{array}{c}\text { Estadios de } \\
\text { Rhodnius prolixus }\end{array}$} & \multicolumn{2}{|c|}{$\begin{array}{l}\text { Infección } \\
\text { por T. cruzi }\end{array}$} \\
\hline & I & II & III & IV & $\mathbf{v}$ & Adulto & Total & $n$ & $\%$ & I & II & III & IV & V & Adulto & Total & $\mathrm{n}$ & $\%$ \\
\hline 1 & - & - & 2 & $1 / 1$ & - & $1 / 1$ & 4 & 2 & 50,0 & - & 1 & $2 / 2$ & $2 / 2$ & - & $1 / 1$ & 6 & 5 & 83,3 \\
\hline 2 & - & - & $3 / 2$ & $5 / 2$ & - & $4 / 3$ & 12 & 7 & 58,3 & - & $3 / 1$ & $2 / 2$ & $3 / 2$ & $1 / 1$ & $4 / 3$ & 13 & 9 & 62,2 \\
\hline 3 & - & 1 & $1 / 1$ & $2 / 1$ & 1 & 3 & 8 & 2 & 25,0 & - & - & - & $4 / 1$ & - & $5 / 2$ & 9 & 3 & 33,3 \\
\hline 4 & - & - & - & $1 / 1$ & - & $1 / 1$ & 2 & 2 & 100,0 & - & - & $2 / 2$ & $1 / 1$ & - & - & 3 & 3 & 100,0 \\
\hline 5 & - & - & 1 & 2 & $1 / 1$ & - & 4 & 1 & 25,0 & - & - & $1 / 1$ & $3 / 3$ & - & $1 / 1$ & 5 & 5 & 100,0 \\
\hline 6 & - & - & - & $2 / 1$ & $2 / 2$ & 1 & 5 & 3 & 60,0 & - & - & $2 / 1$ & $1 / 1$ & $1 / 1$ & $3 / 2$ & 7 & 5 & 71,4 \\
\hline 7 & - & - & $1 / 1$ & $1 / 1$ & - & 2 & 4 & 2 & 50,0 & - & - & 1 & 2 & - & $3 / 2$ & 6 & 2 & 33,3 \\
\hline 8 & 2 & 1 & $3 / 1$ & $8 / 6$ & - & $4 / 2$ & 18 & 9 & 50,0 & - & - & 3 & $3 / 2$ & - & $4 / 2$ & 10 & 4 & 40,0 \\
\hline 9 & - & - & 1 & $2 / 2$ & $2 / 1$ & $3 / 1$ & 8 & 4 & 50,0 & - & 1 & $1 / 1$ & $1 / 1$ & $2 / 1$ & 2 & 7 & 3 & 42,9 \\
\hline 10 & - & - & - & $4 / 2$ & 1 & $1 / 1$ & 6 & 3 & 50,0 & - & - & - & $1 / 1$ & $1 / 1$ & $3 / 3$ & 5 & 5 & 100 \\
\hline 11 & - & - & 1 & 1 & - & $2 / 1$ & 4 & 1 & 25,0 & - & 2 & $1 / 1$ & $3 / 2$ & $2 / 2$ & $4 / 2$ & 12 & 7 & 58,3 \\
\hline 12 & - & - & $2 / 2$ & 2 & - & $1 / 1$ & 5 & 3 & 60,0 & - & 2 & - & - & $1 / 1$ & - & 3 & 1 & 33,3 \\
\hline 13 & - & - & - & - & - & - & - & - & - & - & - & - & - & - & - & - & - & - \\
\hline 14 & - & - & - & - & - & - & - & - & - & - & - & - & - & - & - & - & - & - \\
\hline Total & 2 & 2 & $15 / 7$ & $31 / 17$ & $7 / 4$ & $23 / 12$ & 80 & 39 & $43,1^{*}$ & - & 9 & $15 / 12$ & $24 / 12$ & $8 / 12$ & $30 / 12$ & 86 & 52 & $54,1^{*}$ \\
\hline
\end{tabular}

In= número de ejemplares positivos para Trypanosoma cruzi en el estadio

No se reportaron triatominos (-)

* valor medio de la proporción 


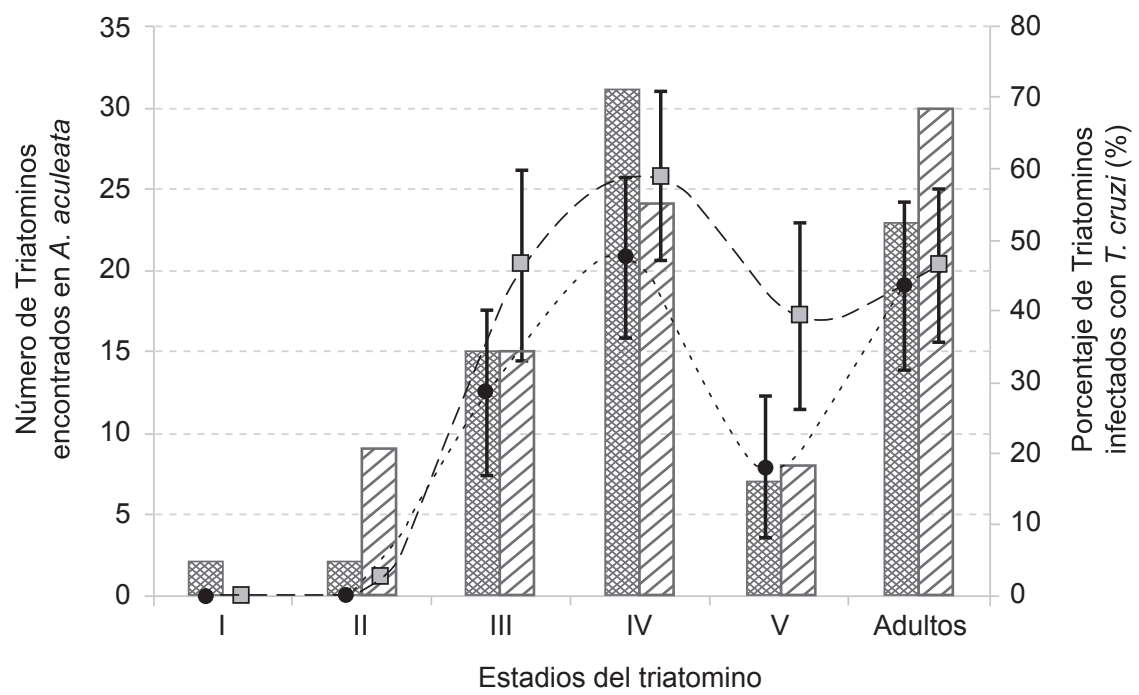

Número de Triatoma maculata

In Número de Rhodnius prolixus

- \% de T. maculata infectado con T. cruzi

$\neg-\%$ de R. prolixus infectado con T. cruzi

Figura 2. Frecuencia de infestación de triatominos y su porcentaje de infección por Trypanosoma cruzi discriminada por estadios ontogénicos colectados en ejemplares de Acrocomia aculeata del estado Anzoátegui, Venezuela

El material de contenido intestinal del $10 \%$ los triatominos positivos para cada especie, produjo en el modelo murino una infección con periodo prepatente promedio de 17 días, parasitemia máxima promedio de $2 \times 10^{5}$ flagelados $/ \mathrm{mL}$ de sangre y recuperación de los aislados por xenodiagnóstico en $75 \%$ de los 12 ejemplares utilizados para cada especie, con formación de metacíclicos a razón de $24 \%$ en promedio de los flagelados del contenido intestinal visualizados en fresco, en un intervalo de 30 a 45 días posinfección y con capacidad infectante.

El contenido intestinal de los triatominos que presentaron flagelados, al ser analizado con el marcador ADNk para T. cruzi, mediante amplificación por PCR y electroforésis en gel de agarosa, mostró la presencia de la banda de 330 pb, en un $95 \%$ de los ejemplares (Figura 3), lográndose
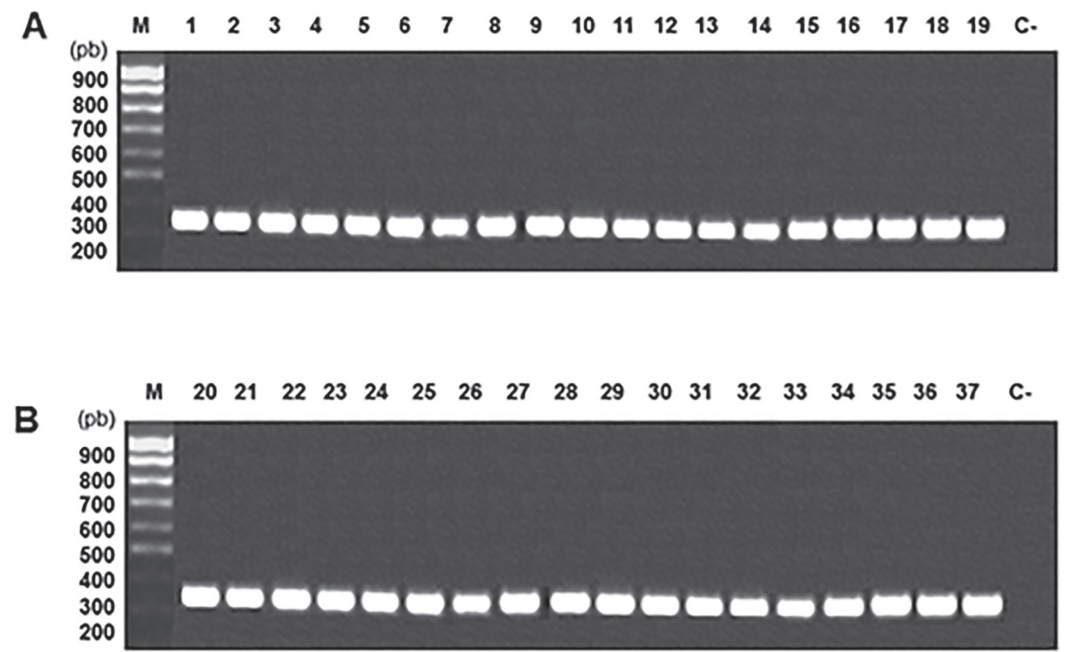

Figura 3. Electroforesis en agarosa al $1 \%$ de los productos de reacción de ADNk para aislados de Trypanosoma cruzi a partir de heces de triatominos colonizando Acrocomia aculeata del Estado Anzoátegui, Venezuela. A) (M) Marcador de 100 pb (Promega), (1) aislado de T. cruzi Dm28 (control positivo) (2-19) muestras de heces de triatominos (Rhodnius prolixus) colonizando Acrocomia aculeata, (C-) control negativo. B) (M) Marcador de $100 \mathrm{pb}$ (Promega), (20) aislado de Trypanosoma cruzi Dm28 (control positivo), (21-37) muestras de heces de triatominos (Triatoma maculata) colonizando Acrocomia aculeata, (C-) control negativo 
identificar a los flagelados como estadios de T. cruzi tanto parasitológica como molecularmente.

La caracterización de los aislados de $T$. cruzi a través de la amplificación de la región intergénica del gen de mini-exón; de la fracción $24 S a$ y $18 \mathrm{~S}$ del gene ribosomal, mostraron un perfil Tcl caracterizado por bandas de 350, 110 y 175 pb respectivamente, el patrón de amplificación fue similar a aquel exhibido para aislados de referencias $\mathrm{Tcl}$, aislado Dm28c (MDID/VE/1984/Dm28c) incluido como control (Figura 4).

\section{DISCUSIÓN}

Los ejemplares de $A$. aculeata desafectados (a menos de 500 metros de la vivienda) en campañas epidemiológicas de saneamiento fitosanitario y considerados en este estudio, fueron explorados en épocas del año de poca variación climatológica diaria y anual, caracterizada por baja precipitación. Este hecho y la vitalidad de su follaje, parecieron proporcionar un ambiente húmedo adecuado, altamente correlacionado con la presencia y el desarrollo de estadios adultos de las dos especies de triatominos, $R$. prolixus y $T$. maculata que se encontraron, los cuales comparten hábitat con invertebrados, vertebrados poiquilotermos y homotermos, estos dos últimos referidos como fuente sanguínea para los triatominos vectores ${ }^{(16) .}$

Se amplió el estudio hasta determinar la infección por $T$. cruzi de las dos especies de triatominos encontradas, reflejando ambas especies de triatominos un incremento progresivo hacia los estadios más próximos al adulto (estadio alado único capaz de desplazarse mediante el vuelo hacia habitats domésticos) a excepción del quinto estadio que se mostró disminuido en número, susceptible a predadores e inanición ${ }^{(16)}$, lo cual favorecería el rol de estos insectos como vectores en la enfermedad de Chagas.

Los aislados de T. cruzi presentes en este estudio conformaron el genotipo Tcl (predominante en Venezuela) revelando subpoblaciones homogéneas del parásito con poco intercambio, quizás por ser un hábitat cerrado ${ }^{(17)}$.

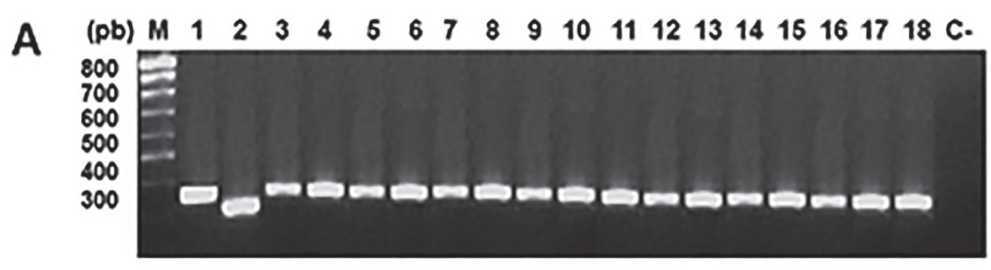

(pb) M $\begin{array}{lllllllllllllllllll}1 & 2 & 3 & 4 & 5 & 6 & 7 & 8 & 9 & 10 & 11 & 12 & 13 & 14 & 15 & 16 & 17 & 18 & \mathrm{C} .\end{array}$
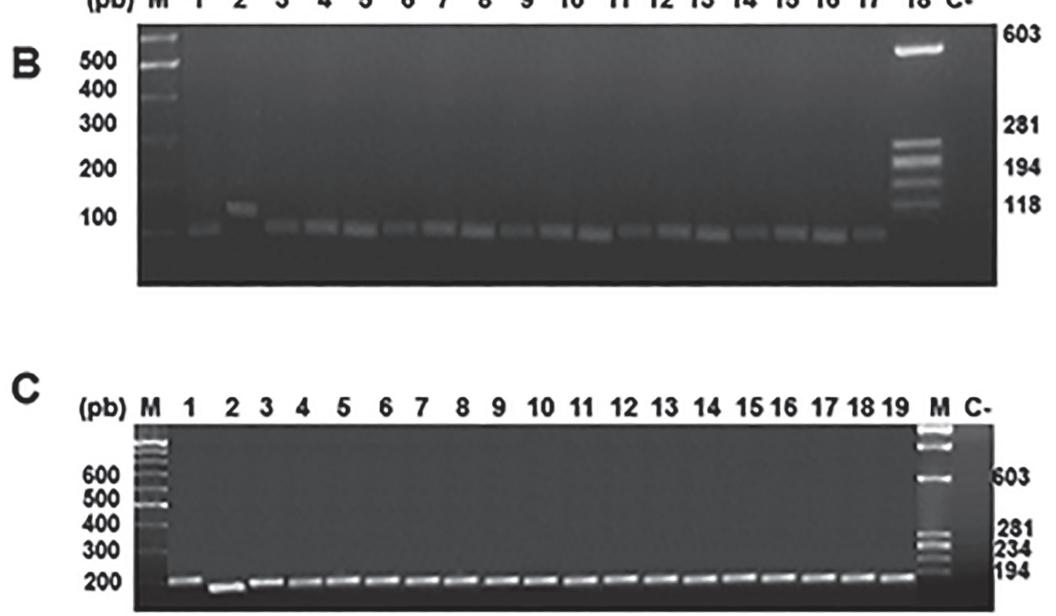

Figura 4. Caracterización molecular de los aislados de Trypanosoma cruzi obtenidos de Acrocomia aculeata. A) Amplificación del espaciador no transcrito del gen del miniexón. (M) Marcador de tamaño molecular 100 pb (Promega), (1) Control positivo (Tcl), (2) Control positivo (TcV), (3-18) ADN extraídos de muestras de heces de triatominos. B) Amplificación de la subunidad 24Sa del DNA ribosomal. (M) Marcador de tamaño molecular 100 pb (Promega), (1) Control positivo (Tcl), (2) Control positivo (TcV), (3-17) ADN extraídos de muestras de heces de triatominos. C) Amplificación de la subunidad $18 \mathrm{~S}$ del ADN ribosomal, (M) Marcador de tamaño molecular 100 pb (Promega), (1) Control positivo (Tcl), (2) Control positivo (TcV), (3-19) ADN extraídos de muestras de heces de triatominos 
El bajo número de palmeras reportadas se debió a que se aprovechó una desafectación manejada por las autoridades regionales, más que una intervención propia sobre los ejemplares, en campañas profilácticas. La presencia de $T$. cruzi en los triatominos, no sería suficiente para asegurar directamente que las especies estén participando en el ciclo de transmisión enzoótica. Sin embargo, la existencia de palmas corozo de tan larga duración (media de 17 años) con un follaje adecuado para el desarrollo de todos los estadios ontogénicos de estos insectos, asegurarían una regular y continua presencia de triatominos infectados y un riesgo epidemiológico en el estado Anzoátegui.

La presencia de los huevos de estos triatominos en las celdillas de las pencas, en las bases y en la totalidad de las hojas, utilizados para la construcción de techos y paredes de la vivienda humana sería otro elemento favorecedor de la dispersión y domiciliación de estos potenciales vectores ${ }^{(2,5,9)}$.

La distribución de los triatominos está afectada por su capacidad de explotar un amplio rango de hábitats cuando sus nidos ecológicos son dispersados, favoreciéndose que algunas especies invadan ambientes domésticos, comenzando así la zoonosis ${ }^{(15)}$.

Los triatominos pueden desplazarse cientos de metros y ser atraídos por la luz artificial, moviéndose desde ambientes peridomiciliares o silvestres tan endógenos, como acúmulos de piedra, árboles huecos, madrigueras de animales o inclusive palmas, hasta la vivienda humana ${ }^{(16,18)}$.

La colonización de triatominos en palmeras ha sido referida en la cuenca amazónica brasileña, Llanos Orientales y costa caribeña de Colombia, en Corrientes, Argentina y en la zona del canal de Panamá (19-24).

La simpatría de diferentes especies de triatominos en palmas como en este estudio, ya había sido citada por Freitas et al. ${ }^{(25)}$ quienes encontraron a Rhodnius neglectus, Triatoma sordida y Panstrongylus megistus en palma corozo de Brasil, asociados a murciélagos y didelfideos, infectados con T. cruzi.

En Venezuela se ha referido infestación triatomínica en 427 palmeras de varias especies, tales como Copernicia tectorum, Mauritia minor, Mauritia flexuosa, Attalea humboldtiana, Oenocarpus batana, Leopoldina piassaba, Jasennia batana, Jasenia policarpa y A. aculeata, la referida palma corozo, de nuestro interés. En esta se ha registrado $46 \%$ de infestación, con hasta 1999 ejemplares de $R$. prolixus, de los cuales un total de 224 (160 ninfas y 64 adultos), mostraron un índice de infección por T. cruzi y $T$. rangeli de $12,9 \%$ y de $1,3 \%$, respectivamente ${ }^{(9)}$.

Longa y Scorza ${ }^{(19)}$ evidenciaron la infestación de 629 ejemplares de palma corozo por Rhodnius robustus en el estado Trujillo en Venezuela, de los cuales 32,9\%, se mostraron infectados por T. cruzi, considerándose la dispersión de éste triatomino desde las palmeras hacia los domicilios cercanos, un evento de riesgosa frecuencia.

A esto se suma, el estudio en el norte del estado Anzoátegui de 14 cocoteros (Cocos nucifera), ejemplares de alto valor en cosmética y alimentación, como hábitats de $R$. prolixus y T. maculata y avanzan por primera vez en la presencia en palma corozo, de estas dos especies de triatominos como cohabitantes junto con potenciales predadores como podrían ser los escorpiones ${ }^{(2,4)}$.

Los focos naturales de tripanosomiasis americana son tan variados como los hábitats de sus reservorios y la marcada euritopía y euritrofía de los vectores, lo cual les confiere ubicuidad. Con base a estos resultados estaríamos frente a la palma corozo como un hábitat, con elementos para una potencial zoonosis en el área de estudio.

Las palmeras han tenido variados usos; de la palma corozo se utiliza el mesocarpio del fruto, para consumo fresco o en la industria para la fabricación de harina, elaboración artesanal y/o industrial de helados, mermeladas y vinos. Las semillas son utilizadas para la extracción de aceite de uso culinario y las raíces se utilizan en medicina tradicional como hipotensor. En particular, el cogollo se hierve para la elaboración de harinas y el licor de savia, conocido como vino de coyol o champaña llanera (9). La transmisión por vía oral de $T$. cruzi ha producido brotes importantes de la enfermedad de Chagas, como los ocurridos en Brasil por consumo de jugo de caña de azúcar contaminada con restos de triatominos ${ }^{(26)}$.

En Venezuela se presentó uno de los brotes mas grandes de enfermedad de Chagas, ocurrido en escolares por transmisión oral a través del consumo de jugo de frutas contaminados con material de triatominos ${ }^{(31,32)}$, seguido de brotes de transmisión oral por consumo de jugos de frutas en escolares de Chichiriviche de La Costa (estado Vargas), en la parroquia Antímano del Distrito Capital; y el brote más reciente en el estado Táchira ${ }^{27-29)}$

Estos antecedentes alertan sobre el consumo del fruto de la palma corozo y del licor producido a partir de su estípite e incluso el manejo de la palmera en su totalidad, lo cual junto a la cercanía de las palmas a la vivienda humana, constituyen factores de riesgo para la contaminación por T. cruzi.

La adaptación palmera-triatomino revelaría que estos grupos vegetales parecen ser hábitats naturales poco intervenidos en los cuales se presentan los elementos bióticos como poblaciones estables de triatominos que favorecerían la circulación del agente etiológico de la enfermedad de Chagas en un nivel de microhábitat no antrópico, con capacidad de 
dispersarse mediante el uso de elementos como las hojas de palma y sus frutos y así formar parte de los focos endémicos de la zoonosis ${ }^{(5)}$

Dado que los triatominos silvestres, generalmente no pueden ser totalmente controlados por químicos en la reinfestación e invasión de la vivienda humana, su estudio y vigilancia es el principal desafío a largo plazo para el control sostenido de la enfermedad de Chagas en las Américas ${ }^{(5,30)}$.

En conclusión, se reportó la infestación de $A$. aculeata o palma corozo con ejemplares triatominos de las especies $R$. prolixus y $T$. maculata en simpatria, infectadas ambas en proporciones similares con sub poblaciones Tcl de T.cruzi, el genotipo más abundante del parásito en Venezuela. Así, A. aculeata pudiese ser un bioindicador de riesgo de la presencia de la enfermedad de Chagas para poblaciones humanas circunvecinas o que pudiesen hacer uso de los frutos y el follaje de estas palmas en regiones del oriente de Venezuela.

Contribución de autoría: AM y LH participaron en la concepción y diseño del artículo; análisis e interpretación de datos; redacción del artículo; EF participó en el desarrollo de pruebas, análisis e interpretación de datos y revisión crítica del artículo; RB, MV y JDCH participaron en la recolección del material de estudio recolección de resultados; análisis e interpretación de datos, AR-G participó en la asesoría estadística.

Financiamiento: Ministerio del Poder Popular para la Salud de Venezuela, Proyecto Misión Ciencia en red «Reservorios, Vectores y Participación Ciudadana en las estrategias de prevención y control de la enfermedad de Chagas en áreas endémicas de Venezuela» $N^{\circ}$ 2008000911-6; Proyecto estratégico FONACIT N ${ }^{\circ}$ 2011000470; Proyecto CDCH PG 038171-2011.

Conflictos de interés: Ninguno.

\section{REFERENCIAS BIBLIOGRÁFICAS}

1. World Health Organization. [Internet]. Geneva: WHO; 2017. Chagas Disease (American trypanosomiasis). (2017). Fact sheet 340. Disponible en: http://www.who. int/mediacentre/factsheets/fs340/es/

2. Morocoima A, Chique D, Zavala-Jaspe R, Diaz-Bello Z; Ferrer E, Urdaneta-Morales $\mathrm{S}$, et al. Commercial coconut plant as a natural ecotope of Chagas disease vector in northeastern Venezuela. J Vector Borne Dis. 2010;47(2):76-84.

3. Morocoima A, Chique J, Herrera L, Urdaneta-Morales S. Eratyrus mucronatus (Satl, 1859) (Hemiptera, Reduviidae, Triatominae): primer registro para el estado Anzoátegui (Venezuela). Bol Mal Salud Amb. 2010;50(2):307-10.

4. Morocoima A, De Sousa L, Herrera L, Rojas L, Villalobos M, Chique JD, et al. Simpatría de triatominos (Reduviidae) y escorpiones (Buthidae) en Cocos nucifera y Acrocomia aculeata (Aracaceae) de Anzoátegui, Venezuela. Bol Mal Salud Amb. 2011;51(2):187-98.

5. Abad-Franch F, Lima MM, Sarquis O, Gurgel-Gonçalves R, Sánchez-Martín M, Calzada J, et al. On palms, bugs, and Chagas disease in the Americas. Acta Trop. 2015;151:126-41. doi: 10.1016/j. actatropica.2015.07.005.

6. a-venezuela.com [Internet]. Caracas; 2018 [consulta 6 de julio de 2018]. Mapas viales de Venezuela [1 pantalla]. Disponible en: http://www.a-venezuela. com/index.php/mapas-de-venezuela/ mapas-viales
7. Centro Virtual de Meteorología [Internet]. Venezuela. [Citado el 13 de marzo de 2016]. Disponible en: http://www. meteoven.org/datos.html

8. Instituto Nacional de Estadísticas [Internet]. Caracas: INE; 2018 [consulta 13 de marzo de 2015]. Censo 2011 [1 pantalla]. Disponible en: http://www.ine. gov.ve/CENSO2011

9. Gamboa J. Comprobación de Rhodnius prolixus extradoméstico en Venezuela. Gaceta Med Caracas. 1963;71:205-19.

10. Lent H, Wygodzinsky P. Revision of the Triatominae (Hemiptera: Reduviidae) and their significance as vectors of Chagas' disease. Bull Am Mus Nat Hist. 1979;163:125-520.

11. Herrera L, Urdaneta-Morales S. Avances en la caracterización de biodemes de Trypanosoma (Schizotrypanum) cruzi aislado de urbanizaciones y parques recreacionales del valle de Caracas (Venezuela). Act Biol Venez. 2000;20: 45-51.

12. Ferrer E, Da Conceicão F, Campioli P, Lares M, López M, Rivera MG, et al. Validación de protocolos de PCR para el diagnóstico molecular de la Enfermedad de Chagas. Salus. 2009; 12, Suplemento 1:163-74.

13. Wincker P, Britto C, Pereira JB, Cardoso MA, Oelemann W, Morel CM. Use of a simplified polymerase chain reaction procedure to detect Trypanosoma cruzi in blood samples from chronic Chagasic patients in a rural endemic area. Am J Trop Med Hyg . 1994; 51:771-7.

14. Brisse S, Verhoef J, Tibayrenc M. Characterization of large and small subunit rRNA and miniexon genes further supports the distinction of six Trypanosoma cruzi lineages. Int J Parasitol. 2001;31:1218-26.

15. Patterson JS, Guhl F. Geographical Distribution of Chagas Disease. En: Telleria J, Tibayrenc M, editors. American Trypanosomiasis Chagas Disease. One Hundred Years of Research. 1th Ed. United Kingdom: Elsevier. 2010. p. 54-123.

16. Reyes-Lugo M, Reyes-Contreras M, Salvi I, Gelves W, Avilán A, Llavaneras D, et al. The association of Triatoma maculate (Ericsson 1848) with the gecko Thecadactylus rapicauda (Houttuyn 1782) (Reptilia: Squamata: Gekkonidae): A strategy of domiciliation of the Chagas disease peridomestic vector in Venezuela? Asian Pac J Trop Biomed. 2011;1(4):279-84. doi: 10.1016/S2221-1691(11)60043-9.

17. Carrasco HJ, Segovia M, Morocoima A, Llewellyn MS, Urdaneta-Morales S, Martínez C, et al. Geographical distribution of Trypanosoma cruzi genotypes in Venezuela. PLoS Negl Trop Dis. 2012;6(6):e1707. doi: 10.1371/journal. pntd.0001707.

18. Texeira AR, Monteiro PS, Rebelo JM, Argañaraz ER, Vieira D, Lauria-Pires L, et al. Emerging Chagas disease: Trophic network and cycle of transmission of Trypanosoma cruzi from palm trees in the Amazon. Emerg Infect Dis. 2001; 7:100- 112 .

19. Longa A, Scorza JV. Acrocomia aculeata (Palmae), hábitat silvestre de Rhodnius robustus en el Estado Trujillo, Venezuela. Parasitol Latinoam. 2005; 60:17-24. 
20. Bar ME. Triatominos de la comunidad de palmeras en la provincia de Corrientes. Ecología e importancia epidemiológica [Tesis Doctoral]. Corrientes: Facultad de Ciencias Exactas y Naturales y Agrimensura, Universidad Nacional del Nordeste; 2001.

21. Christensen A, Sousa OE, De Vasquez AM. Host feeding profiles of Triatoma dimidiata in peridomestic habitats of western Panama. Amer J Trop Med Hyg. 1988;38:477-9.

22. Carcavallo R, Susana I, Curto de Casas S, Sherlock I, Galíndez-Girón I, Jurberg $\mathrm{J}$, et al. Geographical distribution and altitudinal dispersion. En: Carcavallo RU, Galíndez-Girón I, Jurberg J, Lent H, editores. Atlas of Chagas Disease Vectors in the Americas. Editora Fiocruz RJ Brasil; 1999. p. 747-92.

23. $\mathrm{D}^{\prime}$ Alessandro A, Barreto-Saravia N, Barreto M. Epidemiology of Trypanosoma cruzi in the Oriental Plains of Colombia. Am J Trop Med Hyg. 1985;33:1084-95.
24. Romaña C, Pizarro J, Rodas E, Guilbert E. Palm trees as ecological indicators of risk areas for Chagas disease. Trans Roy Soc Trop Med Hyg. 1999;93:594-5.

25. De Freitas J, Siqueira A, Ferreira O. Investigações epidemiológicas sobre triatomíneos de hábitos domésticos e silvestres com auxílio da reação de precipitina. Rev Inst Med Trop São Paulo. 1960;2:90-9.

26. Steindel M, Kramer L, Scholl D, Soares M, de Moraes MH, Eger I, et al. Characterization of Trypanosoma cruzi isolated from humans, vectors, and animal reservoirs following an outbreak of acute human Chagas disease in Santa Catarina State, Brazil. Diagn Microbiol Infect Dis. 2008;60(1):25-32. doi: 10.1016/j. diagmicrobio.2007.07.016.

27. Rodriguez-Morales AJ. Chagas disease: an emerging food borne. J Infect Dev Countries. 2008;2:149-50.

28. Noya BA, Díaz-Bello Z, Colmenares C, Ruiz-Guevara R, Murillo L, MuñozCalderón $\mathrm{A}$, et al. Update on oral
Chagas disease outbreaks in Venezuela: epidemiological, clinical and diagnostic approaches. Mem Inst Oswaldo Cruz. 2015;110(3):377-86. doi: 10.1590/007402760140285.

29. Benítez JA, Araújo B, Contreras K, Rivas M, Ramírez P, Guerra W, et al. Urban outbreak of acute orally acquired Chagas disease in Táchira, Venezuela. J Infect Dev Ctries. 2013;7(8):638-41. doi: 10.3855/ jidc. 3620 .

30. Feliciangeli M, Dujardin JP, Bastrenta B, Mazzarri M, Villegas J, Flores M, et al. Is Rhodnius robustus (Hemiptera: Reduviidae) responsible for Chagas disease transmission in Western Venezuela? Trop Med Int Health. 2002;7(3):280-7.

Correspondencia: Leidi Herrera

Dirección: Instituto de Zoología y Ecología Tropical (IZET), Facultad de Ciencias, Av. Los Ilustres. Antigua ETI. Universidad Central de Venezuela (UCV). Caracas, Venezuela. Teléfono: +582126051017 apartado postal 1041-A. Correo electrónico:herrerleidi@gmail.com

\section{Suscríbete a nuestro canal de YOUIIUhe y disfruta la mejor y más completa información de investigación científica} You www.youtube.com/user/RPMESP

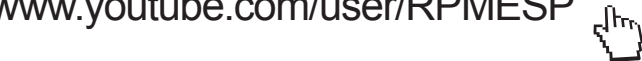

